Check for updates

Cite this: RSC Adv., 2017, 7, 55326

\title{
Mass transfer investigation in a horizontal-vertical pulsed packed extraction column $\uparrow$
}

\author{
Sajad Khooshechin, (D) ${ }^{\text {ab }}$ Mohammad Ali Moosavian, ${ }^{a}$ Jaber Safdari $^{* b}$ \\ and Mohammad Hassan Mallah ${ }^{\mathrm{b}}$
}

In this study, hydrodynamic parameters and the mass transfer coefficient were investigated in a pilot version of a horizontal-vertical pulsed packed extraction column using a toluene-acetone-water system. The effects of the operational parameters on the volumetric overall mass transfer coefficient and hydrodynamic parameters away from the flooding point were studied, and it was found that the volumetric overall mass transfer coefficient, mean drop size and disperse phase holdup were significantly affected by the pulse intensity. However, the dispersed and continuous phase flow rates have a weaker effect. On the other hand, by increasing the pulse intensity the mean drop size and volumetric overall mass transfer coefficient were reduced and increased, respectively, in both sections of the column, but the disperse phase holdup displayed different behavior in the horizontal and vertical sections. By increasing the pulse intensity, the disperse phase holdup was first reduced and then increased in the horizontal section; however, an incremental trend was observed in the vertical section. The effect of the dispersed phase flow rate was significant for all the investigated parameters, but weaker. For instance, all the investigated parameters were increased by enhancing the dispersed phase flow rate. In contrast, the continuous phase flow rate has sometimes had no significant effect, for example, on the mean drop size in both sections of the column. Finally, two empirical correlations for the prediction of the volumetric overall mass transfer coefficient based on the continuous phase were derived in terms of the Sherwood number, Reynolds number and other dimensionless numbers. Good agreement between predictions and experiments was found for all operating conditions that were investigated.

Received 20th July 2017

Accepted 15th November 2017

DOI: $10.1039 / \mathrm{c} 7 \mathrm{ra07999k}$

rsc.li/rsc-advances degree of turbulence imparted to the system and the interfacial area. ${ }^{7}$ The rate of mass transfer in liquid-liquid extraction can be increased by mechanical agitation such as pulsations imparted to the liquids by an external mechanical or electronic device. Internal mechanical parts are eliminated, leakage is minimized, and the pulsator can be isolated..$^{7-10}$

One form of countercurrent extraction equipment that uses an external mechanical device is the pulsed column. Pulsed columns have been of interest in the field of liquid-liquid extraction because pulsing improves the rate of mass transfer by reducing the drop size. ${ }^{10}$ Chantry et al. (1955) showed that the column height required to achieve a given degree of extraction is reduced by a factor of three via pulsation. These columns have an obvious advantage over other mechanical contactors when processing corrosive or radioactive solutions because the pulsing unit can be remote from the column. In addition, the absence of moving mechanical parts in such columns facilitates repair and servicing. On the basis of the published literature, pulsed extraction columns can be categorized into the vertical type (sieve plate, packed, and disc and doughnut), the horizontal type (sieve plate and packed) and the horizontal-vertical type (sieve plate and packed), of which the horizontal-vertical pulsed packed column is introduced in this work.
${ }^{a}$ School of Chemical Engineering, Faculty of Engineering, University of Tehran, Tehran, Iran

${ }^{b}$ Material and Nuclear Fuel Research School, Nuclear Science and Technology Research Institute, P.O. Box: 11365-8486, Tehran, Iran. E-mail: Jsafdari@aeoi.org.ir; Fax: +98 21 88221116; Tel: +982188221117

$\dagger$ Electronic supplementary information (ESI) available. See DOI: 10.1039/c7ra07999k 
Most reported investigations have dealt with the sieve plate and packing type of vertical structures., ${ }^{\mathbf{9 1 1}, \mathbf{1 2}}$ However, there are some reasons why horizontal pulsed columns are more desirable than vertical columns, such as their easier maintenance when throughput is high or higher performance in limited areas. ${ }^{13}$ However, horizontal pulsed columns have a lower production capacity than vertical columns in the same conditions. As both vertical and horizontal columns have their own advantages and disadvantages, it is important to consider the specific process. Regarding the third category, better performance is expected from combining horizontal and vertical columns as a horizontal-vertical structure, in particular when restrictions prevent the use of vertical columns. Akhgar et al. ${ }^{14}$ and Amani et al. ${ }^{15}$ have investigated the hydrodynamic parameters of a horizontalvertical pulsed sieve plate column, but no data are published in the literature regarding the mass transfer performance of this structure, even that of the sieve plate. An investigation of the hydrodynamic parameters, as well as the mass transfer performance, is therefore necessary for this packing type.

In order to design or scale up a pulsed column for a desired extraction process, it is essential to predict the mass transfer behavior using a suitable model. There are many approaches for dealing with mass transfer, e.g., the plug flow model (PFM), axial dispersion model (ADM), drop population balance model (DPBM) and forward mixing model (FM). The first two models (PFM and ADM) are widely used for extraction columns. ${ }^{12}$ However, the development of the other models has been rather limited owing to the complexity of the problems involved.

In the present work, for the first time the mass transfer performance and hydrodynamic parameters of a horizontalvertical pulsed packed extraction column were investigated. The volumetric overall mass transfer coefficient was measured using the axial dispersion model to evaluate the performance of the column. The effects of the pulse intensity and the flow rates of the disperse and continuous phases on the hydrodynamic parameters and the volumetric overall mass transfer coefficient were investigated using a toluene-acetone-water system. This is a standard system for evaluating the performance of a liquid-liquid extraction column that has been used by many researchers. ${ }^{9,16-20}$

\section{Experimental}

\subsection{Liquid-liquid system}

Three different liquid-liquid systems have been proposed by the European Federation of Chemical Engineering for evaluating the characteristics of a liquid-liquid extraction column. ${ }^{21}$ These systems comprise butanol-water, toluene-water and butyl acetate-water, which cover a wide range of interfacial tensions. Acetone can be used as the solute in mass transfer experiments. Among these systems, toluene-acetone-water is commonly used by many investigators for evaluating liquidliquid extractors, in particular pulsed columns..$^{\mathbf{9 1 6 - 2 0}}$ The liquidliquid system that was studied in this work was therefore toluene-acetone-water. The direction of mass transfer was from the aqueous to the organic phase (continuous to disperse phase). In all experiments, dilute solutions were investigated with approximately $3.5 \mathrm{wt} \%$ acetone in the aqueous phase.
Distilled water was used as the continuous phase, and a technical grade of toluene with a purity of at least $99.5 \mathrm{wt} \%$ was used as the disperse phase. The physical properties of the liquidliquid system at the laboratory temperature are listed in Table 1. All experiments were carried out at a temperature of $20 \pm 1{ }^{\circ} \mathrm{C}$.

\subsection{Description of equipment}

A schematic diagram of the experimental apparatus is shown in Fig. 1. The active part of the column in both horizontal and vertical sections, which was filled with packing to a height of 1.3 $\mathrm{m}$, had an internal diameter of $0.06 \mathrm{~m}$ and was made of glass. Ceramic rushing rings with a diameter of $0.63 \mathrm{~cm}$ and a porosity of $60 \%$ were used as packing. Five pairs of samplers were arranged at equal intervals along the effective zones of the horizontal and vertical sections of the column. The location of the interface of the two phases at the top of the column was automatically controlled by an optical sensor. A solenoid valve that was normally kept closed was positioned in the outlet stream of the continuous phase. This valve received electronic signals from the optical sensor. When the location of the interface was about to change, the optical sensor sent a signal to the solenoid valve and the aqueous phase was allowed to leave the column. The organic phase was allowed to leave the column via an overflow. The contents of the column were pulsed at the desired amplitude and frequency using compressed air that was frequently forced into an air leg joined to the bottom settling zone of the column.

\subsection{Procedure}

Before the experiments were carried out, both phases were saturated, after which the solute was added to the continuous phase to give an acetone concentration of about $3.5 \mathrm{wt} \%$. The amplitude and frequency of the pulsations were next adjusted to the desired values and, after the column had been filled with the continuous phase, the disperse phase was introduced. In the steady-state condition samples were withdrawn from sampling valves installed on the horizontal and vertical sections. After a sample was taken, up to $5 \mathrm{~mL}$ of the aqueous phase was immediately separated, and the solute concentration was determined using a UV/vis spectrophotometer analyzer.

The drop size was measured during steady-state conditions and determined using a photographic technique. Digital photos were taken using a Nikon D5000 digital camera. The drop size was measured at three positions in the active zones of the column in both horizontal and vertical sections. The drop

Table 1 Physical properties of studied system at $20^{\circ} \mathrm{C}$ (ref. 21)
Physical property

$\rho_{\mathrm{c}}\left(\mathrm{kg} \mathrm{m}^{-3}\right)$

$\rho_{\mathrm{d}}\left(\mathrm{kg} \mathrm{m}^{-3}\right)$

$\mu_{\mathrm{c}}$ (mPpa s)

$\mu_{\mathrm{d}}$ (mPpa s)

$\sigma\left(\mathrm{mN} \mathrm{m}^{-1}\right)$

$D_{\mathrm{c}}\left(\mathrm{nm}^{2} \mathrm{~s}^{-1}\right)$

$D_{\mathrm{d}}\left(\mathrm{nm}^{2} \mathrm{~s}^{-1}\right)$
Toluene-acetone-water

$994.5-995.7$

$864.4-865.2$

$1.059-1.075$

$0.574-0.584$

$27.5-30.1$

$1.09-1.14$

$2.7-2.8$ 


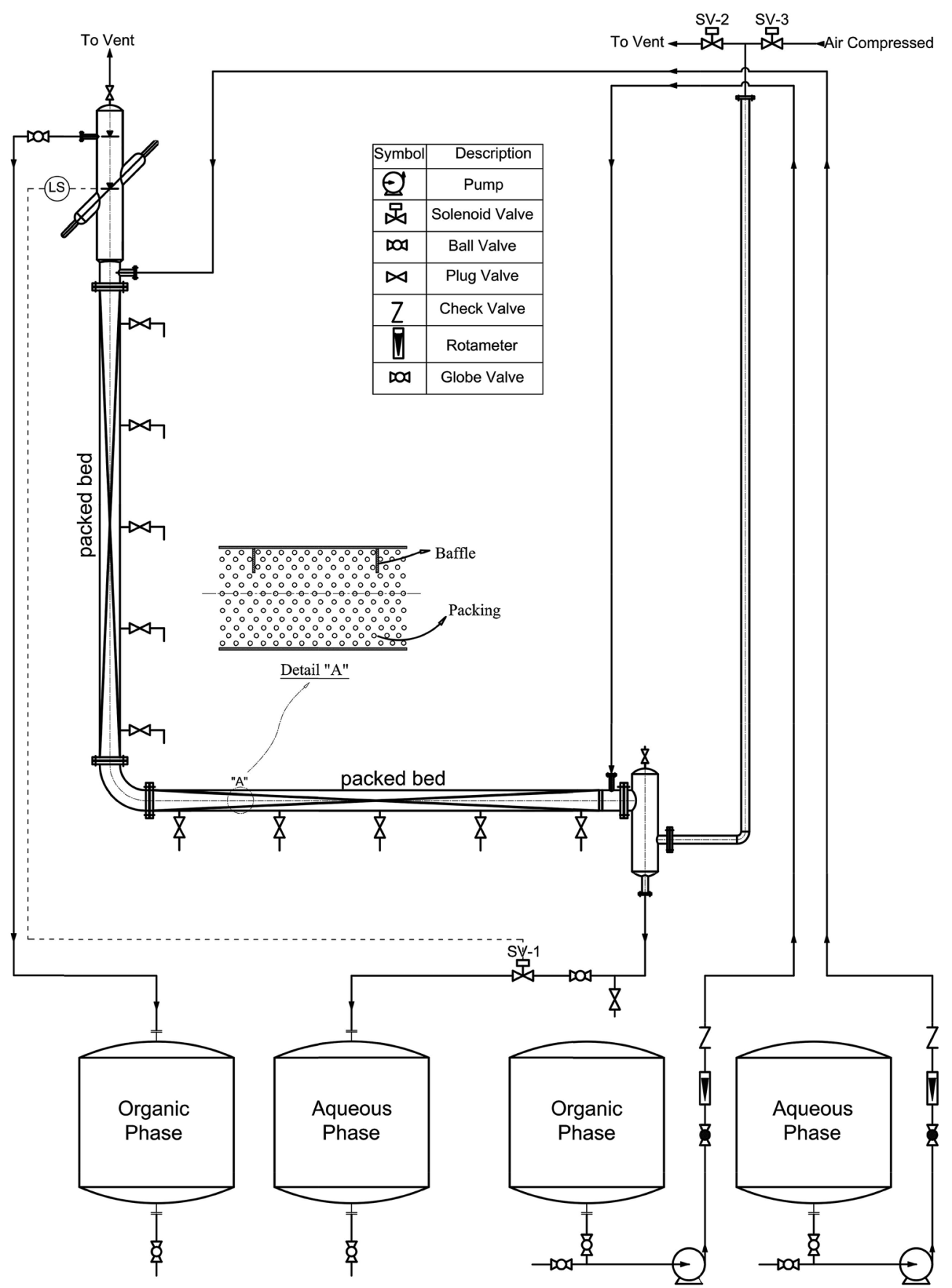

Fig. 1 Schematic diagram of the horizontal-vertical pulsed packed extraction column.

dimensions were then analyzed by AutoCAD software. More than 1500 drops were selected for each set of experimental conditions at six points in the active zones (three points in each section) to guarantee that the Sauter mean drop diameter that was determined had statistical significance. The Sauter mean diameter $d_{32}$ was calculated using the following equation:

$$
d_{32}=\frac{\sum_{i=0}^{n} n_{i} d_{i}{ }^{3}}{\sum_{i=0}^{n} n_{i} d_{i}^{2}}
$$

where $n_{i}$ is the number of droplets with a mean diameter of $d_{i}$ within the narrow size range $i$.

The disperse phase holdup $(\varphi)$ is defined as the volume fraction of the extraction column that is occupied by the disperse phase:

$$
\varphi=\frac{v_{\mathrm{d}}}{v_{\mathrm{c}}+v_{\mathrm{d}}}
$$

where $v_{\mathrm{d}}$ and $v_{\mathrm{c}}$ are the volumes of the disperse and continuous phases, respectively.

In the present work, three operational parameters, namely, the pulse intensity and the volumetric flow rates of both phases, 
were examined. Twenty experiments were carried out according to the central composite design method (CCDM), whereby the parameters were systematically varied in 15 experiments with the central data point repeated six times in order to determine the accuracy of the system in question and check the reproducibility of the experiments, which proved to be good. The error of the system was found to be about $4.3 \%$. The intervals of the pulse intensity and the volumetric flow rates of the continuous and disperse phases were set to $1.2-1.6 \mathrm{~cm} \mathrm{~s}^{-1}$, 1.0-4.0 $\mathrm{L} \mathrm{h}^{-1}$, and 1.0-4.0 $\mathrm{L} \mathrm{h}^{-1}$, respectively.

The average absolute relative deviation (AARD) was used to compare the predicted results with the experimental data, and is defined as follows:

$$
\operatorname{AARD}=\left(\frac{1}{\mathrm{NDP}}\right) \sum_{i=1}^{\mathrm{NDP}} \frac{\mid \text { experimental value }- \text { predicted value } \mid}{\text { experimental value }}
$$

where NDP is the number of data points.

\section{Modelling}

As mentioned before, there are many different approaches for dealing with mass transfer in liquid-liquid extraction columns. Among these models, the axial dispersion model combines relative ease of implementation with the achievement of relatively accurate results. ${ }^{11}$ Therefore, this model was selected for the present study.

The simplest approach for dealing with mass transfer is based on the ideal assumption that each phase moves in perfect plug flow and all elements of both phases have the same residence time in the column. The column wall and internals cause fluids to flow in all directions, which invalidates the above assumption. To overcome this defect, Danckwerts (1953) for the first time proposed that for each phase all the known and unknown influencing parameters that cause deviations from ideal flow could be amalgamated into one parameter, which is named the axial dispersion coefficient $(E)$, for the flow of each phase. ${ }^{22}$ On the basis of the axial dispersion model and the mass balance in the column, the equation set for steady-state processes under constant boundary conditions for both phase velocities was established as follows:

$$
\begin{gathered}
\frac{1}{\mathrm{Pe}_{\mathrm{c}}} \frac{\mathrm{d}^{2} x}{\mathrm{~d} Z^{2}}-\frac{\mathrm{d} x}{\mathrm{~d} Z}-N_{\mathrm{oc}}\left(x-x^{*}\right)=0 \\
\frac{1}{\mathrm{Pe}_{\mathrm{d}}} \frac{\mathrm{d}^{2} y}{\mathrm{~d} Z^{2}}+\frac{\mathrm{d} y}{\mathrm{~d} Z}+N_{\mathrm{oc}} \frac{u_{\mathrm{c}}}{u_{\mathrm{d}}}\left(x-x^{*}\right)=0
\end{gathered}
$$

where $N_{\mathrm{oc}}=K_{\mathrm{oc}} a H / u_{\mathrm{c}}$, which represents the number of mass transfer units, and $\mathrm{Pe}_{\mathrm{c}}$ and $\mathrm{Pe}_{\mathrm{d}}$ are the respective Peclet numbers, which can be defined as $\mathrm{Pe}_{\mathrm{c}}=H u_{\mathrm{c}} / E_{\mathrm{c}}$ and $\mathrm{Pe}_{\mathrm{d}}=H u_{\mathrm{d}} / E_{\mathrm{d}} . E_{\mathrm{c}}$ and $E_{\mathrm{d}}$ represent the axial dispersion coefficients for the flow of the continuous and disperse phases, respectively. $K_{\mathrm{oc}} a$ is the volumetric overall mass transfer coefficient based on the continuous phase. The following open-open boundary conditions were used for the boundary condition, which required the solution of sets of second-order equations: ${ }^{12,16,23}$

$$
\begin{aligned}
& Z=0, \rightarrow x=x_{\text {in }} \text { and } y=y_{\text {out }} \\
& Z=1, \rightarrow x=x_{\text {out }} \text { and } y=y_{\text {in }}
\end{aligned}
$$

\section{Results and discussion}

\subsection{Hydrodynamics}

As mentioned in most reference works, a pulsed column can operate in three different flow regimes, i.e., mixer-settler, dispersion and emulsion, which depend on the pulse intensity $\left(A f=\right.$ amplitude $\times$ frequency). ${ }^{10}$ The mixer-settler regime is characterized by the separation of light and heavy phases into discrete and clear layers during the quiescent period of the pulse cycle. The dispersion regime is the next in sequence and is found upon a further increase in the pulse intensity. This regime is characterized by a non-uniform drop size distribution

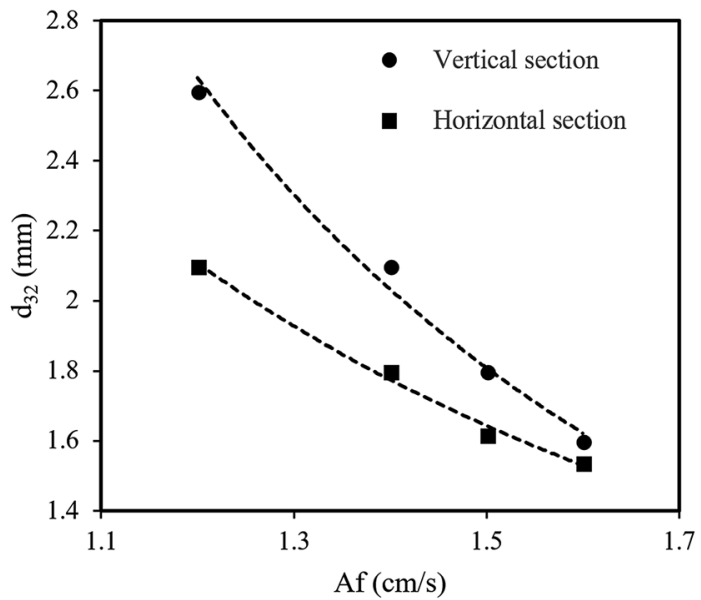

(a)

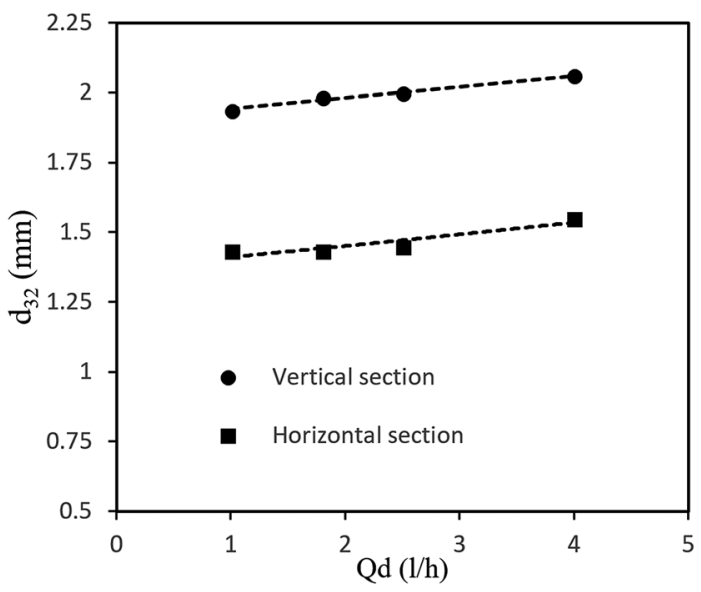

(b)

Fig. 2 (a) Effect of the pulse intensity on the mean drop size at constant dispersed and continuous phase flow rates of $2.5 \mathrm{~L} \mathrm{~h}^{-1}$. (b) Effect of the dispersed phase flow rates on the mean drop size at a constant continuous phase flow rate and pulse intensity of $2.5 \mathrm{~L} \mathrm{~h}^{-1}$ and $1.4 \mathrm{~cm} \mathrm{~s}^{-1}$, respectively. 
and no coalescence of drops of the disperse phase. With an increase in the pulse velocity, the drop size decreases and thus an emulsion is formed. All these flow regimes can be observed in the vertical and horizontal types of pulsed sieve plate column. However, on the basis of our knowledge and practical observations, only the dispersion and emulsion regimes are found in a vertical pulsed packed column, in contrast to the horizontal type. Regarding the structure of the pulsed column used in this work, we should determine the transitional pulse intensity required to work in the dispersion regime. This is usually the recommended regime, as it combines high flux and good mass transfer. ${ }^{24}$ Therefore, as was described above, it was possible to identify visually different flow regimes by applying different pulse velocities. Thus, values of $A$ and $f$ that led to the dispersion regime were selected. After a few tests, the minimum and maximum pulse intensities for working in the dispersion regime were found to be 1.2 and $1.6 \mathrm{~cm} \mathrm{~s}^{-1}$, respectively. The maximum intensity was chosen to prevent flooding.

4.1.1. Sauter mean diameter. The drop size has an important effect on the disperse phase holdup and the maximum throughput. Furthermore, the interfacial mass transfer area can be determined using a combination of drop size and holdup. ${ }^{25}$ The drop size obviously depends on the rates of drop breakage and coalescence. On the basis of the experiments conducted in this research, the main operational parameters that affect the mean drop size are the pulse intensity and the flow rates of the disperse phase. The effect of the pulse intensity on the Sauter mean drop size is shown in Fig. 2a. As can be seen, the Sauter mean drop size decreased with an increase in the pulse intensity, because drop breakage is enhanced with an increase in pulse intensity owing to an increase in collisions between dispersed liquid drops and the internal wall. ${ }^{26}$ Another important point that can be seen from Fig. 2a is that the Sauter mean drop size in the vertical section is larger than in the horizontal section. However, this difference was reduced by increasing the pulse intensity.

The experimental results revealed that the volumetric flow rates had a weaker effect than the pulse intensity on the drop size. As seen in Fig. 2b, the mean drop size slightly increased with the increase in the dispersed phase flow rate. The coalescence rate of drops increased owing to the increase in the flow rate of the disperse phase so that the mean drop size increased. Moreover, in this research, a change in the flow rate of the continuous phase had no significant effect on the mean drop size. It should be noted that the dotted lines in the figures are only guides to the eye. As shown above, the mean drop size is mostly affected by the pulse intensity and flow rate of the disperse phase, and contours of the mean drop size upon changes in the pulse intensity and flow rate of the disperse phase are shown in Fig. 3.

4.1.2. Dispersed phase holdup. As well as the mean drop size, the disperse phase holdup is another essential parameter for the design and scale-up of columns. Values of the holdup can be used to determine the flooding velocity, and it is entirely related to the interfacial area available for mass transfer. The effect of the pulse intensity on the disperse phase holdup is illustrated in Fig. 4a. As can be seen, in the vertical section the disperse phase holdup increased on an increase in the pulse intensity; however, it exhibited two different behaviors in the horizontal section. The velocity of moving drops has three

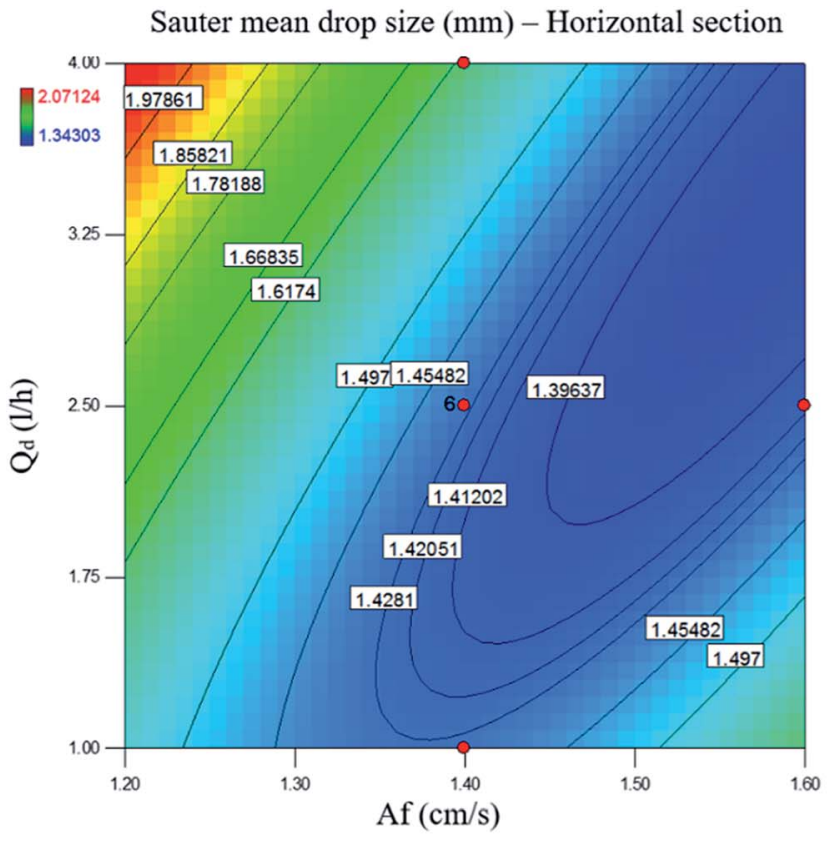

(a)

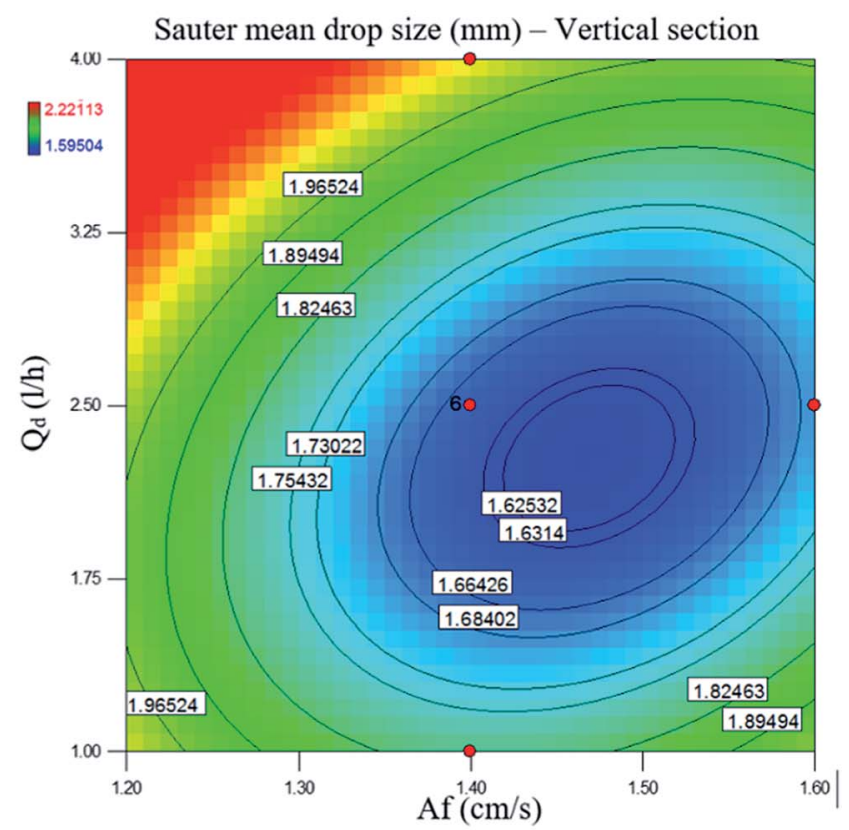

(b)

Fig. 3 Contours of the mean drop size versus the pulse intensity and dispersed phase flow rate at a constant continuous phase flow rate of $2.5 \mathrm{~L} \mathrm{~h}^{-1}$ : (a) horizontal section; (b) vertical section. 


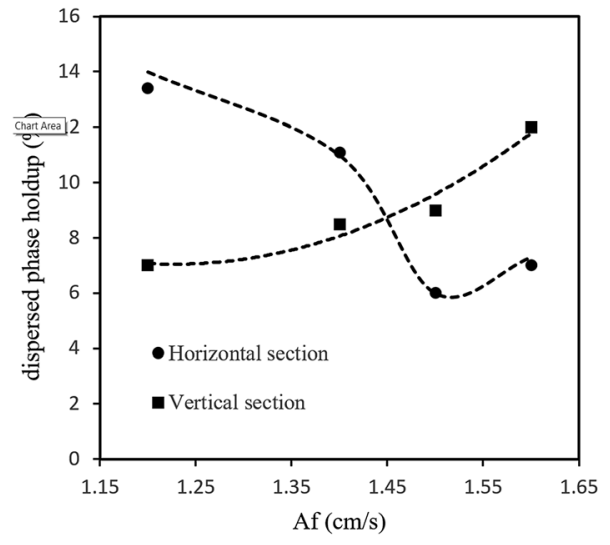

(a)

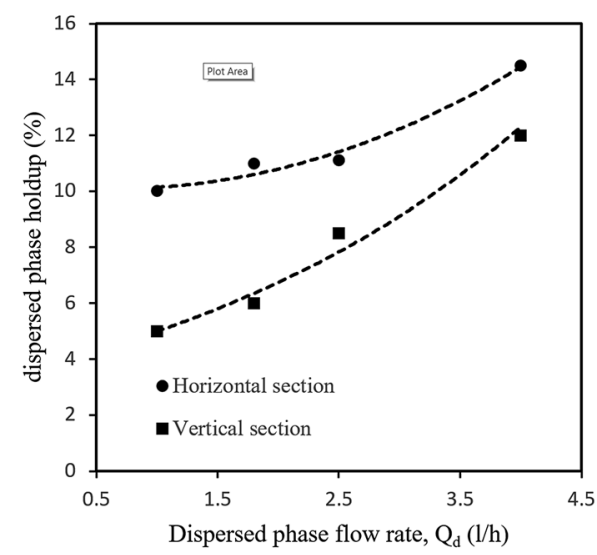

(b)

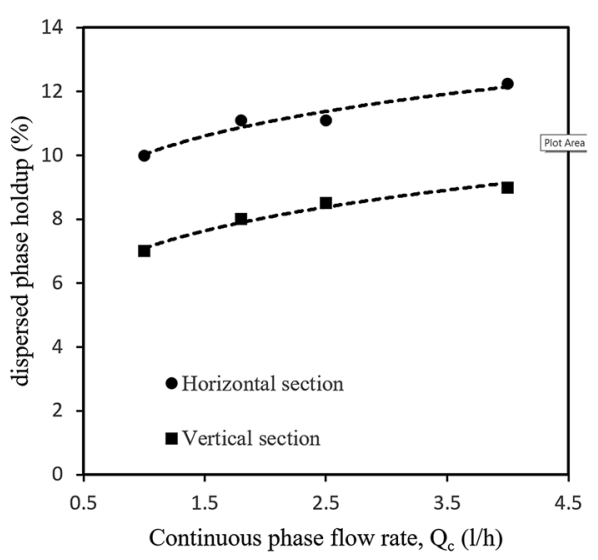

(c)

Fig. 4 (a) Effect of the pulse intensity on the disperse phase holdup at constant continuous and dispersed phase flow rates of $2.5 \mathrm{~L} \mathrm{~h}^{-1}$. (b) Effect of the dispersed phase flow rate on the dispersed phase holdup at a constant continuous phase flow rate and pulse intensity of $2.5 \mathrm{~L} \mathrm{~h}^{-1}$ and $1.4 \mathrm{~cm} \mathrm{~s}^{-1}$, respectively. (c) Effect of the continuous phase flow rate on the dispersed phase holdup at a constant dispersed phase flow rate and pulse intensity of $2.5 \mathrm{~L} \mathrm{~h}^{-1}$ and $1.4 \mathrm{~cm} \mathrm{~s}^{-1}$, respectively.

components: a permanent component due to the flow rate, a pulse component due to the pulsations and a buoyancy component. ${ }^{27}$ The third component is only important in the vertical section. When the pulse intensity increases the pulse component of the drop velocity increases and the buoyancy component decreases because the drop size is smaller. Experimental results revealed that the impact of the buoyancy component was greater than that of the pulse component in the vertical section, because the holdup was increased by increasing the pulse intensity, which implies that the drop velocity in the vertical section decreased. However, an increase in the pulse intensity caused an increase in the velocity of drops in the horizontal section, and therefore the disperse phase holdup decreased. With a further increase in the pulse intensity, the holdup declined and reached a minimum value. As the upper flooding limit was approached, the holdup significantly increased and the smallest drop fractions were washed out by the continuous phase.

The effects of the flow rates of the disperse and continuous phases on the holdup are shown in Fig. $4 \mathrm{~b}$ and c. It is clearly observed from these figures that the disperse phase holdup increased upon increasing the flow rate of the disperse phase in both horizontal and vertical sections. However, the flow rate of the continuous phase had a slight effect on the holdup. As with the mean drop size, the disperse phase holdup is also mostly affected by the pulse intensity and flow rate of the disperse phase, and contours of the disperse phase holdup upon changes in the pulse intensity and flow rate of the disperse phase are shown in Fig. 5.

\subsection{Mass transfer}

According to work carried out by other investigators, ${ }^{9,12,16}$ the main operational parameters that affect the mass transfer performance of a column are the pulse intensity and flow rate of the disperse phase. Fig. 6a illustrates the effect of the pulse intensity on the volumetric overall mass transfer coefficient. It is seen from Fig. 6a that the mass transfer performance was improved by increasing the pulse intensity in both horizontal and vertical sections. By increasing the pulse intensity, the degree of turbulence imparted to the system is greatly increased. Furthermore, the specific area available for mass transfer is increased because the drop size is smaller, and therefore the overall mass transfer coefficient is increased. As can be seen from Fig. 6a, the overall mass transfer coefficient was higher in the horizontal section. This may relate to the mass transfer direction. The solvent in this extraction research was the disperse phase. Furthermore, the maximum driving force of mass transfer and hence the maximum performance are found where the solvent is pure, because the solvent enters the horizontal section, which has the maximum driving force, and this section thus has the highest mass transfer coefficient. The effect of the flow rate of the disperse phase on the volumetric overall mass transfer coefficient is shown in Fig. 6b. As can be seen, the volumetric overall mass transfer coefficient was slightly increased by increasing the flow rate of the disperse phase. An increase in the velocity of the disperse phase causes an increase in the disperse phase holdup, as well as the drop size, and it is therefore expected that the interfacial area is not changed, but, as demonstrated by Asadollahzadeh et al. ${ }^{28}$ the effect of the disperse phase holdup is 


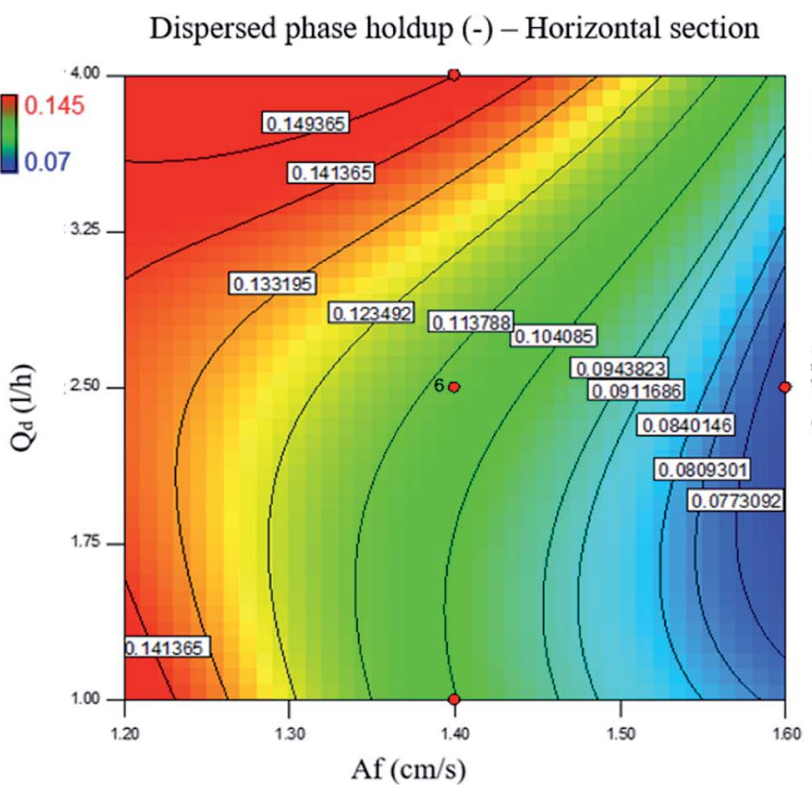

(a)

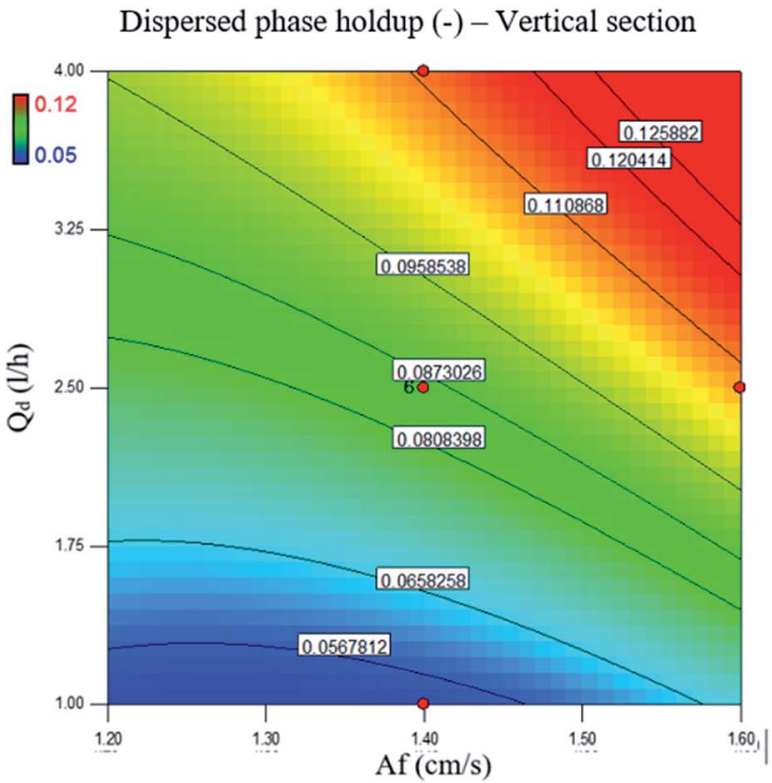

(b)

Fig. 5 Contours of the disperse phase holdup versus the pulse intensity and dispersed phase flow rate at a constant continuous phase flow rate of $2.5 \mathrm{~L} \mathrm{~h}^{-1}$ : (a) horizontal section; (b) vertical section.

more profound and consequently the interfacial area is slightly increased by increasing the velocity of the disperse phase.

\subsection{Predictive correlation of the overall mass transfer coefficient based on the continuous phase}

The development of a new correlation for predicting the overall mass transfer coefficient based on the continuous phase in a horizontal-vertical pulsed packed extraction column is one of the main purposes of the present work. Most correlations that have been proposed for predicting the mass transfer coefficient in an extraction column relate to columns other than pulsed packed columns such as pulsed sieve plate, Kühni, rotating disc and spray columns. However, Torab-mostaedi and Safdari ${ }^{9}$ proposed semiempirical correlations for predicting the Sherwood number of the continuous phase in a pulsed packed extraction column. For the mass transfer direction from the continuous to the disperse phase, they presented the following equation for $10<\operatorname{Re}<150:^{9}$

$$
\mathrm{Sh}_{\mathrm{oc}}=-49.76+14.8 \mathrm{Re}^{0.64}
$$

It should be noted that the overall mass transfer coefficient can be expressed as a dimensionless Sherwood number (see the abovementioned correlation) based on the continuous phase, which is defined as follows:

$$
\mathrm{Sh}_{\mathrm{oc}}=\frac{K_{\mathrm{oc}} d_{32}}{D_{\mathrm{c}}}
$$

In addition, Safari et al. have investigated the mass transfer performance in a pulsed packed column and proposed the following correlation for predicting the overall mass transfer coefficient: ${ }^{12}$

$$
\frac{K_{\text {oc }} a d_{32}}{\rho_{\mathrm{c}} u_{\text {slip }}}=1.9 \times 10^{-8} \frac{\operatorname{Re}^{1.78}}{(1-\varphi)^{2}}\left(\frac{A f}{u_{\text {slip }}}\right)^{0.09}\left(\frac{u_{\mathrm{d}}}{u_{\mathrm{c}}}\right)^{1.62}
$$

Fig. 7 shows a comparison of the experimental value of the Sherwood number in the horizontal section with those calculated by eqn (8) and (10) in the same experimental conditions. As can be seen, the data predicted by both correlations display remarkable deviations from the experimental values, and furthermore a greater deviation is seen for the vertical section. This deviation is mostly due to the structure used in this study. In addition, experimental data for the overall mass transfer coefficient were compared with those obtained by the correlations that were proposed for other types of extraction column, which are presented in Table $\mathrm{S} 1 \uparrow^{\mathbf{9 , 1 2 , 1 6 , 1 9 , 2 9 - 3 5}}$ A reliable correlation for predicting the mass transfer coefficient in a horizontalvertical pulsed packed extraction column is therefore required.

Consequently, the experimental data for the overall mass transfer coefficient based on the continuous phase are correlated in terms of dimensionless numbers. The significance of each dimensionless number for the overall mass transfer coefficient has been investigated using statistical methods. Finally, the following equations were obtained for predicting the overall mass transfer coefficients in the horizontal and vertical sections, respectively:

$$
\begin{aligned}
& \operatorname{Sh}_{\mathrm{oc}}(\mathrm{H})=0.00224 \mathrm{Re}_{\mathrm{H}}{ }^{3.44}\left(\frac{A f}{U_{\text {slip }}}\right)^{1.35}\left(\frac{u_{\mathrm{c}}}{u_{\mathrm{d}}}\right)^{1.31} \\
& \mathrm{Sh}_{\mathrm{oc}}(\mathrm{V})=2 \times 10^{-5} \mathrm{Re}_{\mathrm{V}}{ }^{5.29}\left(\frac{A f}{U_{\text {slip }}}\right)^{4.95}\left(\frac{u_{\mathrm{c}}}{u_{\mathrm{d}}}\right)^{1.19}
\end{aligned}
$$




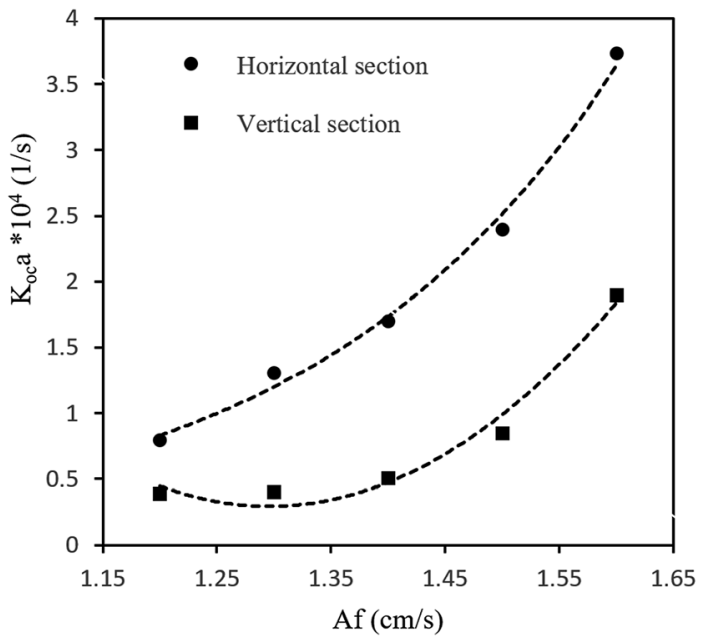

(a)

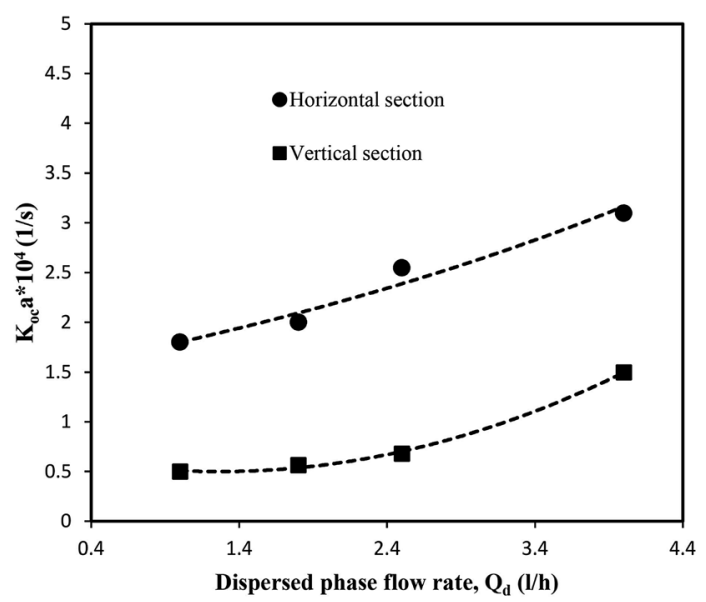

(b)

Fig. 6 (a) Effect of the pulse intensity on the volumetric overall mass transfer coefficient at constant continuous and dispersed phase flow rates of $2.5 \mathrm{~L} \mathrm{~h}^{-1}$. (b) Effect of the dispersed phase flow rate on the volumetric overall mass transfer coefficient at a constant continuous phase flow rate and pulse intensity of $2.5 \mathrm{~L} \mathrm{~h}^{-1}$ and $1.4 \mathrm{~cm} \mathrm{~s}^{-1}$, respectively.

where Re is the well-known Reynolds number, $A f$ is the pulse intensity, $u_{\mathrm{c}}$ and $u_{\mathrm{d}}$ are the superficial velocities of the continuous and disperse phases, respectively, and $U_{\text {slip }}$ is the slip (or relative) velocity between two phases through the column. Re and $\mathrm{U}_{\text {slip }}$ are calculated as follows:

$$
\begin{gathered}
\mathrm{Re}=\frac{\rho_{\mathrm{c}} U_{\text {slip }} d_{32}}{\mu_{\mathrm{c}}} \\
U_{\text {slip }}=\frac{Q_{\mathrm{c}}}{\varepsilon A(1-\varphi)}+\frac{Q_{\mathrm{d}}}{\varepsilon A \varphi}
\end{gathered}
$$

where $d_{32}$ is the Sauter mean diameter, $\rho_{\mathrm{c}}$ and $\mu_{\mathrm{c}}$ are the density and viscosity of the continuous phase, respectively, $\varphi, \varepsilon$ and $A$ are the disperse phase holdup, packing porosity and crosssectional area of the column, and finally $Q_{\mathrm{c}}$ and $Q_{\mathrm{d}}$ are the volumetric flow rates of the continuous and disperse phases, respectively.

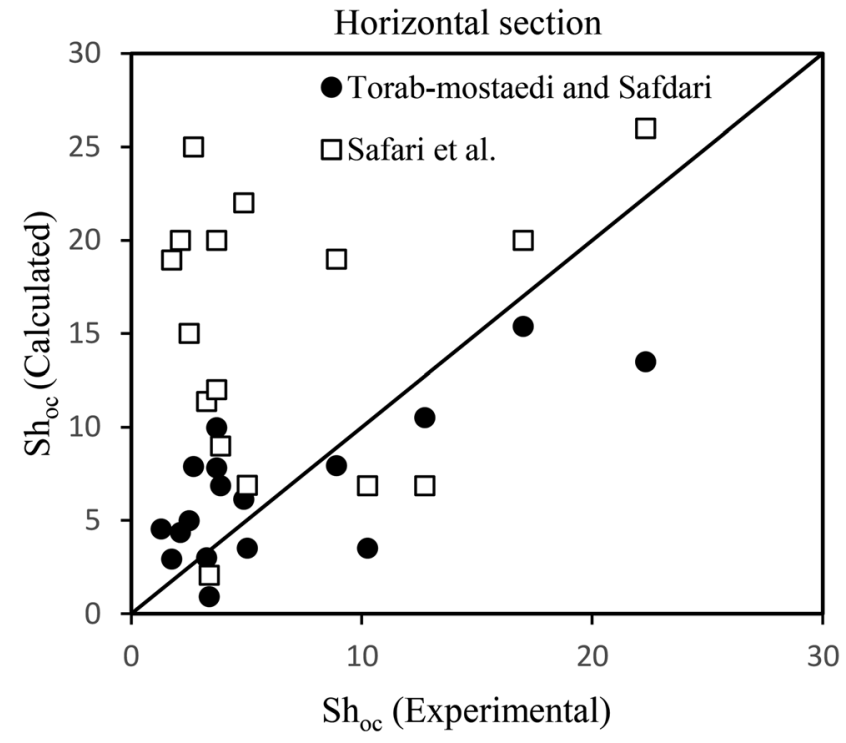

Fig. 7 Comparison of experimental values of the Sherwood number with values calculated using the equations proposed by Torab-mostaedi and Safdari ${ }^{9}$ and Safari et al. $^{12}$ for the water-acetone-toluene system.

In order to evaluate the overall mass transfer coefficients predicted using eqn (11) and (12), experimental data were compared with those predicted by eqn (11) and (12). The average relative deviations (ARDs) of the values of $\mathrm{Sh}_{\mathrm{oc}}(\mathrm{H})$ and $\mathrm{Sh}_{\mathrm{oc}}(\mathrm{V})$ predicted using eqn (11) and (12) from experimental data were $12.1 \%$ and $15.5 \%$, respectively.

A comparison of the experimental data with values calculated by eqn (11) and (12) is illustrated in Fig. 8. It can be seen that most of the predicted values have an error of less than $20 \%$, except at two and three points in the horizontal and vertical

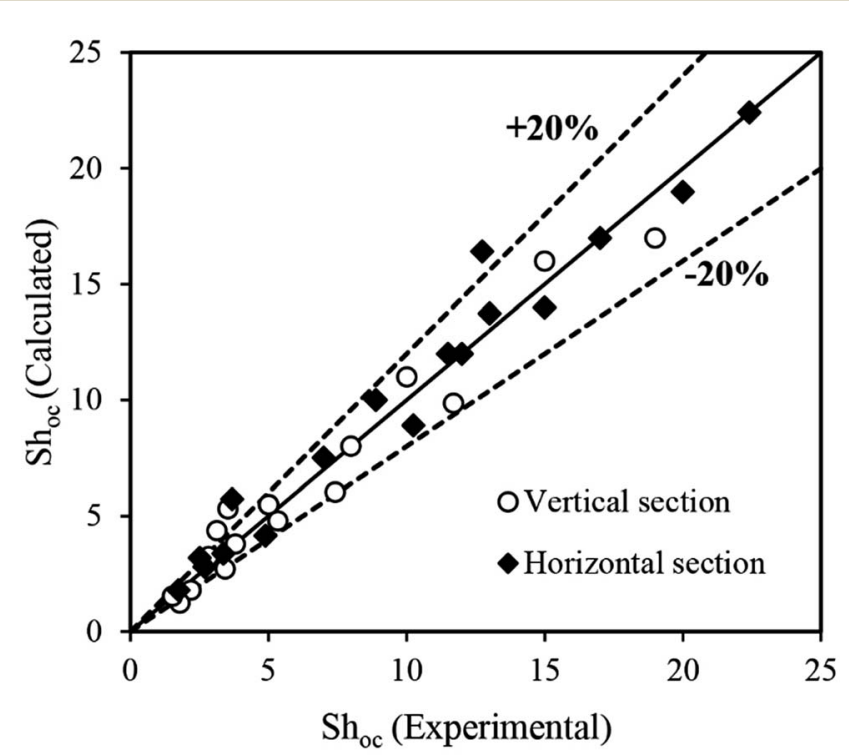

Fig. 8 Comparison of experimental data with values calculated using eqn (11) and (12). 
Table 2 Axial dispersion coefficients of the continuous and disperse phases on an increase in the pulse intensity ${ }^{a}$

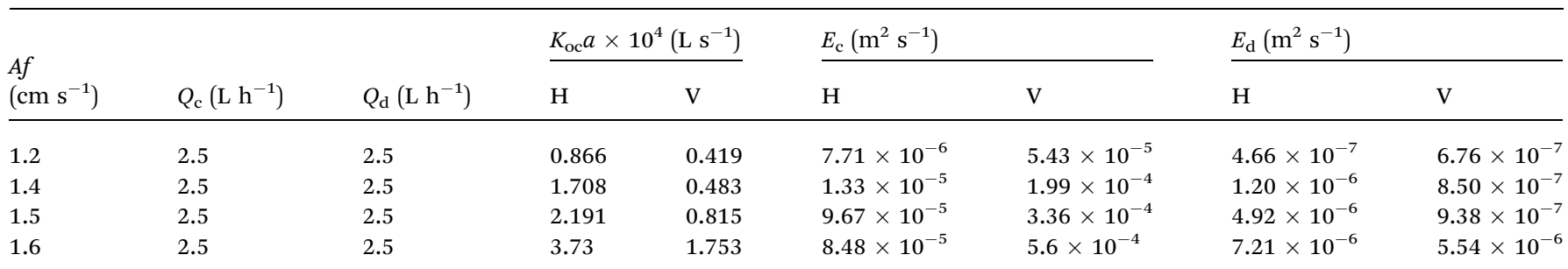

${ }^{a} \mathrm{H}$ : horizontal section, V: vertical section.

sections, respectively. According to Fig. 8, there is good agreement between the experimental and calculated data.

\subsection{Axial dispersion coefficients of continuous and disperse phases}

The axial dispersion coefficient represents all the known and unknown influencing parameters that cause deviations from plug flow. ${ }^{22}$ Therefore, a lower axial dispersion coefficient is more favorable, because non-ideal terms reach a minimum and the mass transfer performance can be improved. ${ }^{36}$ As shown by Amani et $a .^{23}$ and other investigators, the pulse intensity has the greatest impact on axial dispersion, and the effects of the two other operational parameters are negligible.,9,16,23,37 Table 2 shows the effect of the pulse intensity on the axial dispersion coefficients of the continuous and disperse phases. It is seen that the axial dispersion coefficients of both the disperse and the continuous phase increase with the pulse intensity, which is not suitable for mass transfer performance. In contrast, an increase in pulse intensity leads to an increase in the mass transfer coefficient, and it can be concluded that the axial dispersion effect is not dominant. Thus, the overall result of an increase in pulse intensity is an improvement in the mass transfer performance. Another point observed from Table 2 is that the axial dispersion coefficient of the disperse phase is lower than that of the continuous phase in each section.

\section{Conclusions}

This paper presents an experimental investigation of the mass transfer performance and hydrodynamic parameters of the toluene-acetone-water system in a pilot version of a horizontalvertical pulsed packed extraction column. The investigation of mass transfer was performed using the axial dispersion model. The effects of operational variables, such as the flow rates of the disperse and continuous phases and pulse intensity, on the volumetric overall mass transfer coefficients and hydrodynamic parameters were studied. It was found that the pulse intensity is the most important operational parameter. By increasing the pulse intensity the hydrodynamic parameters changed in such a way that the mass transfer coefficient increased. In addition, the mass transfer coefficient was influenced by the flow rate of the disperse phase. Finally, this work has provided two valuable correlations for the prediction of the volumetric overall mass transfer coefficient in a horizontal-vertical pulsed packed extraction column. Good agreement between predictions and experimental data causes these correlations to be recommended for the calculation of mass transfer coefficients and consequently for the final selection of the column height.

\section{Conflicts of interest}

There are no conflicts to declare.

\section{Nomenclature}

a Interfacial area $\left(\mathrm{m}^{2} \mathrm{~m}^{-3}\right)$

A Amplitude of pulsations (m) or cross-sectional area of the column $\left(\mathrm{m}^{2}\right)$

$f \quad$ Frequency of pulsations $\left(\mathrm{s}^{-1}\right)$

$D \quad$ Molecular diffusivity $\left(\mathrm{m}^{2} \mathrm{~s}^{-1}\right)$

d Drop diameter

$d_{32} \quad$ Sauter mean drop diameter $(\mathrm{m})$

$v \quad$ Phase volume

E $\quad$ Axial mixing coefficient $\left(\mathrm{m}^{2} \mathrm{~s}^{-1}\right)$

$H \quad$ Effective height of the column (m)

$K \quad$ Overall mass transfer coefficient $\left(\mathrm{m} \mathrm{s}^{-1}\right)$

$\begin{array}{ll}\mathrm{Pe} & \text { Peclet number }(\mathrm{Pe}=H u / E) \\ \mathrm{Pe}_{\mathrm{c}} & \text { Peclet number of the continuous phase }\left(\mathrm{Pe}_{\mathrm{c}}=\frac{d_{32} V_{\mathrm{s}}}{D_{\mathrm{c}}}\right)\end{array}$

$N_{\mathrm{oc}} \quad$ Number of 'true' transfer units $\left(N_{\mathrm{oc}}=K_{\mathrm{oc}} a H / u_{\mathrm{c}}\right)$

$Q \quad$ Flow rate of the continuous or dispersed phase $\left(\mathrm{m}^{3} \mathrm{~s}^{-1}\right)$

Re Reynolds number $\left(\rho u_{\mathrm{s}} d_{\mathrm{p}} / \mu\right)$

$\mathrm{Sh}_{\mathrm{oc}} \quad$ Sherwood number based on overall mass transfer coefficient of the continuous phase

$u \quad$ Superficial velocity $\left(\mathrm{m} \mathrm{s}^{-1}\right)$

$U_{\text {slip }} \quad$ Slip velocity $\left(\mathrm{m} \mathrm{s}^{-1}\right)$

$x \quad$ Mass fraction of acetone in the continuous phase

$x^{*} \quad$ Equilibrium mass fraction of acetone in the continuous phase corresponding to the disperse phase Mass fraction of acetone in the disperse phase

\section{Greek symbols}

$\rho \quad$ Density $\left(\mathrm{kg} \mathrm{m}^{-3}\right)$

$\varphi \quad$ Disperse phase holdup

$\mu \quad$ Viscosity $\left(\mathrm{kg} \mathrm{m}^{-1} . \mathrm{s}\right)$

$\varepsilon \quad$ Void fraction

$\sigma \quad$ Interfacial tension between two phases $\left(\mathrm{N} \mathrm{m}^{-1}\right)$ 


\section{Subscripts/superscripts}

c Continuous phase or column

d Disperse phase

o Overall value

* Equilibrium value

in Inlet to the column

out Outlet from the column

\section{References}

1 X. Sun, H. Luo and S. Dai, Chem. Rev., 2012, 112, 2100-2128.

2 S. Saha, R. Mistri and B. C. Ray, J. Chromatogr. A, 2010, 1217, 307-311.

3 S. Saha, R. Mistri and B. C. Ray, J. Chromatogr. A, 2009, 1216, 3059-3063.

4 D. P. Mantuano, G. Dorella, R. C. A. Elias and M. B. Mansur, J. Power Sources, 2006, 159, 1510-1518.

5 H. G. Schutze, W. A. Quebedeaux and H. Lochte, Ind. Eng. Chem., Anal. Ed., 1938, 10, 675-677.

6 J. C. Godfrey and M. J. Slater, Liquid-liquid extraction equipment, Wiley Chichester, UK, 1994.

7 S. Khooshechin, J. Safdari, M. A. Moosavian and M. H. Mallah, Int. J. Heat Fluid Flow, 2013, 44, 684-691.

8 A. Hussain, T.-B. Liang and M. Slater, Chem. Eng. Res. Des., 1988, 66, 541-554.

9 M. Torab-Mostaedi and J. Safdari, Chem. Eng. Process., 2009, 48, 1321-1326.

10 R. Yadav and A. Patwardhan, Chem. Eng. J., 2008, 138, 389415.

11 A. E. Ferreira, S. Agarwal, R. M. Machado, M. L. F. Gameiro, S. M. Santos, M. T. A. Reis, M. R. C. Ismael, M. J. N. Correia and J. M. Carvalho, Hydrometallurgy, 2010, 104, 66-75.

12 A. Safari, J. Safdari, H. Abolghasemi, M. Forughi and M. Moghaddam, Chem. Eng. Res. Des., 2012, 90, 193-200.

13 A. Melnyk, S. Vijayan and D. Woods, Can. J. Chem. Eng., 1992, 70, 417-425.

14 S. Akhgar, J. Safdari, J. Towfighi, P. Amani and M. H. Mallah, $R S C A d v ., 2017,7,2288-2300$.

15 P. Amani, J. Safdari, H. Abolghasemi, M. H. Mallah and A. Davari, Int. J. Heat Fluid Flow, 2017, 65, 266-276.
16 M. Asadollahzadeh, A. Hemmati, M. Torab-Mostaedi, M. Shirvani, A. Ghaemi and Z. Mohsenzadeh, Chin. J. Chem. Eng., 2017, 25, 53-61.

17 M. Torab-Mostaedi, A. Ghaemi, M. Asadollahzadeh and P. Pejmanzad, Braz. J. Chem. Eng., 2011, 28, 447-456.

18 M. Torab-Mostaedi and J. Safdari, Braz. J. Chem. Eng., 2009, 26, 685-694.

19 M. Torab-Mostaedi, S. Safdari, M. Moosavian and M. G. Maragheh, Braz. J. Chem. Eng., 2008, 25, 473-481.

20 M. Torab-Mostaedi, A. Ghaemi and M. Asadollahzadeh, Can. J. Chem. Eng., 2012, 90, 1570-1578.

21 T. Míšek, R. Berger and J. Schröter, EFCE Publ. Ser., 1985, 46, 1.

22 P. Danckwerts, Chem. Eng. Sci., 1953, 2, 1-13.

23 P. Amani, J. Safdari, A. Gharib, H. Badakhshan and M. H. Mallah, Prog. Nucl. Energy, 2017, 98, 71-84.

24 B. Grinbaum, Solvent Extr. Ion Exch., 2006, 24, 795-822.

25 A. Kumar and S. Hartland, Ind. Eng. Chem. Res., 1996, 35, 2682-2695.

26 S. Ousmane, M. Isabelle, M.-S. Mario, T. Mamadou and A. Jacques, Chem. Eng. Res. Des., 2011, 89, 60-68.

27 G. Angelov and C. Gourdon, Chem. Eng. Res. Des., 2012, 90, 877-883.

28 M. Asadollahzadeh, S. Shahhosseini, M. Torab-Mostaedi and A. Ghaemi, Chem. Eng. Res. Des., 2015, 100, 104-112.

29 A. Rahbar-Kelishami and H. Bahmanyar, Chem. Eng. Res. Des., 2012, 90, 615-621.

30 C.-H. He, Y.-H. Gao, S.-H. Yang and D. W. Edwards, J. Loss Prev. Process Ind., 2004, 17, 195-204.

31 A. Kumar and S. Hartland, Chem. Eng. Res. Des., 1999, 77, 372-384.

32 A. F. Seibert and J. R. Fair, Ind. Eng. Chem. Res., 1988, 27, 470-481.

33 R. Clift, Bubbles, drops, and particles, 1978.

34 M. E. Weber, Ind. Eng. Chem. Fundam., 1975, 14, 365-366.

35 A. Lochiel and P. Calderbank, Chem. Eng. Sci., 1964, 19, 471484.

36 T. C. Lo, M. H. I. Baird and C. Hanson, Handbook of solvent extraction, Wiley, New York, 1983.

37 F. Panahinia, J. Safdari, M. Ghannadi-Maragheh, P. Amani and M. H. Mallah, Sep. Sci. Technol., 2017, 52, 1537-1552. 